\title{
Síntomas de ansiedad y depresión en adolescentes escolarizados de 10 a 17 años en Chía, Colombia
}

\section{Symptoms of anxiety and depression in adolescents between 10 to 17 year-old attending schools in Chía, Colombia}

Fanny del Carmen Ospina-Ospina, María F. Hinestrosa-Upegui, María C. Paredes, Yahira Guzmán y Carlos Granados

Universidad de la Sabana. Bogotá, Colombia. fanospina@hotmail.com, nanditahtrosa@hotmail.com, maria.paredes@unisabana.edu.co,yahira.guzman@unisabana.edu.co, caregra@gmail.com

Recibido 23 Octubre 2011/Enviado para Modificación 26 Noviembre 2011/Aceptado 7 Diciembre 2011

\section{RESUMEN}

Objetivo Estimar la prevalencia de síntomas ansiosos y depresivos en adolescentes escolarizados entre los 10-17 años, de la comunidad de Chía Cundinamarca, utilizando las escalas CDI y SCARED, durante los años 2008 a 2010.

Metodología Estudio de corte transversal, realizado en 538 estudiantes. Se identificaron las variables socio demográficas: edad, sexo, seguridad social y centro educativo de procedencia (público, privado, oficial o urbano). Se aplicaron las escalas CDI y SCARED.

Resultados Se detectaron síntomas ansiosos o depresivos en el 40,5\% de la población estudiada (538); de los cuales el $28,3 \%$ presentó síntomas sugestivos de ansiedad exclusivamente, con síntomas depresivos exclusivamente en 3,3 \% y síntomas tanto de ansiedad, como de depresión en 8,9\%. Los síntomas ansiosos fueron más frecuentes en las mujeres y los síntomas depresivos y mixtos se presentaron con más frecuencia en hombres. Se encontró mayor prevalencia de síntomas depresivos $6,9 \%$ en hombres vs $5,4 \%$ en mujeres, dato que no fue estadísticamente significativo.

Conclusión Los síntomas ansiosos y depresivos son frecuentes en adolescentes, se hace indispensable sospecharlos y diagnosticarlos tempranamente, con el fin de poder brindar una atención oportuna. Se deben implementar estrategias que permitan detectar factores protectores y de riesgo para evitar que se desarrollen trastornos mayores, diseñando programas de educación encaminados hacia una buena salud mental de niños y adolescente.

Palabras Clave: Ansiedad, depresión, signos y síntomas, adolescentes (fuente: DeCS, BIREME). 


\section{ABSTRACT}

Objective This study was aimed at estimating the prevalence of symptoms of depression and anxiety amongst 10 to 17 year-old adolescents still attending school using the CDI and SCARED questionnaires for early screening in Chía, a town near Bogota, from 2008 to 2010.

Method This was a cross-sectional study of prevalence.

Results $37 \%$ of the 538 adolescents interviewed had anxiety symptoms, $12.3 \%$ had depression symptoms and $9 \%$ presented anxiety and depression symptoms, males having greater prevalence for depression symptoms $(6.9 \%$ of $5.4 \%$ for females) but lacking statistical significance. There was a greater tendency for anxiety symptoms to be found in adolescents attending public schools. Concerning co morbidity, more anxiety symptoms were found in adolescents having depression symptoms.

Conclusions It was concluded that anxiety and depression symptoms are real at this age, this being reason enough why it is necessary to suspect and detect them on time so that adolescents can receive suitable attention. Protective factors must be strengthened for this to happen and there must be real awareness by everybody to create educational and health programmes directed towards ensuring good mental health.

Key Words: Anxiety, depression, signs and symptoms, adolescent (source: $\mathrm{MeSH}$, $N L M)$.

$O$ e define salud mental como la capacidad de manejar las emociones y lograr vencer obstáculos utilizando herramientas de control y manejo de las situaciones de la vida cotidiana. Cuando esta capacidad se quebranta pueden aparecer alteraciones emocionales y de comportamiento dentro de las cuales se encuentran la ansiedad y la depresión, síntomas que fueron explorados en el presente estudio (1).

El diagnóstico de enfermedad mental, y más si se trata de síntomas depresivos o ansiosos en la población de niños y adolescentes, es un reto para el clínico, dadas las características en ocasiones atípicas de estos cuadros en este grupo de edad. La mayoría de las personas, incluso muchos médicos, consideran que estos síntomas son exclusivos del adulto y que si éstos se encuentran en un niño o en un adolescente, probablemente sean secundarios a "comportamientos" encaminados a llamar la atención o a querer manipular su entorno psicosocial. Existe desconocimiento y desinterés por parte de los profesionales de la salud, padres y profesores, en la identificación temprana de alteraciones mentales y del comportamiento en niños y adolescentes, causando detección tardía de síntomas como 
ansiedad y depresión que pueden terminar en desenlaces como el fracaso académico, conductas suicidas o consumo de sustancias psicoactivas $(1,2)$.

Según la OMS la falta de una adecuada salud mental en las primeras etapas de la vida, puede llevar a trastornos mentales con consecuencias en el largo plazo (3). Así mismo, la Organización Panamericana de la Salud, en América Latina y el Caribe, refiere que cerca de 17 millones de niños padecen trastornos psiquiátricos que requieren intervención sin que estos servicios se les presten (4). La OMS considera que tanto en los países desarrollados como en vías de desarrollo, existe carencia de políticas adecuadas y suficientes, para atender a niños y adolescentes con trastornos emocionales y conductuales $(5,6)$.

Actualmente las alteraciones mentales, que incluyen desórdenes emocionales y del comportamiento, se han convertido en un problema de salud pública, que afecta todos los grupos de edad y se encuentran dentro de las primeras causas de morbimortalidad en la mayoría de los países. La OMS, reporta que 450 millones de personas sufren de estos trastornos, incluidos los relacionados con el consumo de alcohol y el abuso de sustancias psicoactivas.

Estos trastornos afectan a un miembro de una de cada cuatro familias y las proyecciones para el periodo comprendido entre 1990 y 2020 indican que la proporción de la carga mundial de morbilidad correspondiente aumentará hasta un $15 \%$ con respecto al actual (7).

A nivel mundial, 121 millones de personas sufren de depresión, la prevalencia es hasta dos veces más alta en las mujeres que en los hombres (8). La depresión afecta cada vez más a los jóvenes, y como una de sus consecuencias, cada año se suicidan más de 800000 personas, siendo las tasas de suicidio más altas en los últimos años en poblaciones jóvenes, incluyendo niños y adolescentes, tanto para intento de suicidio, como para suicidio consumado (8).

En todo el mundo, cada año 4 millones de adolescentes intentan suicidarse; y de estos mínimo 100000 lo logran (9). La tasa de suicidios varía de un país a otro, los resultados de investigación han mostrado que muchos factores pueden ser responsables por esta variación, dentro de ellos, la estabilidad socioeconómica, la facilidad de acceso a métodos letales y el consumo de alcohol y substancias psicoactivas (3). 
Hasta hace 30 años el National Health Institute (10), incluyó los trastornos de ansiedad y depresión como una entidad psicopatológica que podía ocurrir en edades tempranas. Sin embargo, y a pesar de estar reconocidos como entidades clínicas en niños y adolescentes, se presenta aun falla en el diagnóstico, dado que a pesar de haber síntomas claros de depresión y/o ansiedad, éstos son subestimados por familiares y profesionales de la salud $(3,10,11)$.

Según la OMS, en general, un $20 \%$ de la población infantil y adolescente presenta trastornos mentales, dentro de los cuales se encuentran alteraciones de conducta durante la niñez que tienden a persistir en la adolescencia y adultez. Adicionalmente existen múltiples barreras para el adecuado diagnóstico y tratamiento de estas condiciones, como son el déficit de psiquiatras con formación y experiencia en el manejo de niños y adolescentes, la falta de entrenamiento y ausencia de servicios especializados para niños y adolescentes, lo anterior sumado a la precaria situación económica de algunos países y a la pobre destinación de recursos del estado para una adecuada salud mental (2).

En Colombia, como en el resto del mundo, es una preocupación, el desarrollo afectivo de niños y jóvenes, dado que uno de cada dos presenta alguna sintomatología afectiva, ya sea, leve, moderada o severa, causando disfunción en el vínculo afectivo con familia, profesores y amigos, cosa que se ve reflejada en el deterioro de la calidad de vida y en el funcionamiento global del individuo. En los estudiantes con síntomas afectivos, existe un mayor riesgo de ideación e intentos de suicidio, sin embargo las estrategias de detección y manejo precoz no son suficientes para reducir la frecuencia de dichas conductas y desenlaces fatales (3).

El suicidio en personas jóvenes, especialmente en niños y adolescentes es un fenómeno que viene creciendo desde mediados del siglo XX, llama la atención, no sólo el incremento en las tasas de suicidio en estos grupos de edad, sino también la edad de inicio de dichas conductas, pues se han reportado casos de 6 y 7 años (1). En Colombia, entre los años 2004 y 2006, se hallo un $41 \%$ de ideación suicida y un $16 \%$ de intentos de suicidio en jóvenes de bachillerato entre los 10 y 18 años $(1,2)$. Con este panorama, se hace indispensable la capacitación de profesionales de la salud, docentes y padres de familia en temas relacionados con salud mental, con el fin de que 
éstos adquieran los conocimientos, habilidades y herramientas necesarias como la identificación temprana de signos y síntomas de alarma precisos, que les permitan evitar situaciones de riesgo y actuar adecuadamente, cuando se detecte algún factor de riesgo. La mejor forma de prevenir el desarrollo de complicaciones de trastornos mentales mayores es tener los suficientes conocimientos en diagnóstico, y el entrenamiento adecuado para tomar las conductas pertinentes en el momento indicado (2). En el municipio de Chía, Cundinamarca, según datos sobre el perfil epidemiológico se han reportado casos de violencia intrafamiliar, abandono y deserción escolar, que obedecen principalmente a una situación económica deficiente, pocas oportunidades de capacitación y empleo; y falta de aspiraciones y definición de un proyecto de vida. Esta situación social se refleja en algunas conductas de riesgo en sus jóvenes, como alcoholismo, drogadicción, conformación de pandillas juveniles, a las cuales pertenecen incluso niños desde los 8 años, y actos de vandalismo ejecutados por estas (12).

Los niños y adolescentes con síntomas de ansiedad consultan primero al pediatra y se presentan con miedo o preocupaciones, y por lo general no reconocen su temor como irracional. Comúnmente tienen quejas somáticas de cefalea, dolor de abdominal, llanto, irritabilidad y enojo, que pueden ser mal interpretados como oposición o desobediencia; cuando en realidad representan la expresión del miedo o ansiedad del paciente o el esfuerzo del niño por evitar el estímulo que le causa miedo o ansiedad. Estos síntomas pueden llegar a confundir la evaluación y el diagnostico y llevar a estudios innecesarios. El reconocimiento temprano de los síntomas de ansiedad y la búsqueda inicial de alguna condición médica general que pueda explicar la ansiedad, son de vital importancia para hacer un diagnóstico y tratamiento oportuno y así poder minimizar el impacto negativo $(13,14)$. Existen múltiples escalas y cuestionarios que sirven como tamización de síntomas sugestivos de ansidead y depresión. Unas de las más usadas en diferentes estudios para identificar síntomas sugestivos de depresión es el CDI (Children's Depresión Inventory) y para síntomas sugestivos de ansiedad es el SCARED (Screen for Child Anxiety Related Disorders). 


\section{METODOLOGIA}

Tipo de estudio: Estudio descriptivo de corte transversal.

Población de estudio

Estudiantes adolescentes entre los 10 y 17 años, de colegios ubicados en el municipio de Chía- Cundinamarca.

El municipio de Chía cuenta con 108 colegios distribuidos en no oficial rural, no oficial urbano, oficial rural y oficial urbano. Se excluyeron los colegios preescolares, quedando 99 colegios. Se realizo muestro aleatorio simple de los colegios por estratos y se obtuvo un total de 10 colegios y según el número de estudiantes de cada uno de estos colegios, se determinó proporcionalmente la muestra de estudiantes a encuestar de cada uno de ellos. No oficial rural 3 colegios con una muestra total de 209 alumnos, no oficial urbano 3 colegios con una muestra total de 130 alumnos, oficial rural 2 colegios con una muestra total de 192 alumnos y oficial urbano 2 colegios con una muestra total de 129 alumnos; para un total de 660 alumnos que correspondía a la muestra estimada.

Muestra

Chía contaba con 97444 habitantes y 19500 adolescentes escolarizados, con el rango de edad de interés, durante el período de estudio. La proporción esperada del evento (trastornos mentales y conductuales) fue del $20 \%$, con un nivel de confianza del $95 \%$, error del $3 \%$. Se obtuvo una muestra de 660 escolares, de los cuales se obtuvieron datos completos en 538 escolares, tomándose esta como la muestra final.

Muestreo. Aleatorio simple porque no se pretendía hacer inferencia por colegio o por estratos, si no en la población adolescente escolarizada de Chía, adicionalmente para mejorar la factibilidad del estudio.

Instrumentos

CDI-Children's Depresión Inventory. Creado por Kovacs, 1977-1992, consta de 27 ítems (cognitivos, afectivos y conductuales), para niños entre 8 a 17 años, cada ítem tiene 3 afirmaciones, con un puntaje total 0 a 54, y un punto de corte mayor o igual a 19. S: 97,4 \%, E: 95,6\%, VPP: 0.9 VPN: 0,98 , consistencia interna $0,86(18)$. 
SCARED-Screen for Child Anxiety Related Disorders: creado por Boris Birmaher, consta de 41 ítems, para niños entre 9 a 18 años. Cada ítem tiene 3 afirmaciones, con un puntaje total 0 a 82 , y un punto de corte mayor o igual a 25 . S: $71 \%$, E: $67 \%$. Consistencia interna 0,87 (16,20).

Se realizó un análisis univariado, con variables cuantitativas: Medidas de tendencia central y de dispersión, medidas de frecuencia relativa, con estimación de prevalencias e intervalos de confianza al $95 \%$, tomando como base la población total estudiada. Análisis bivariado, con diferencia de medias mediante la prueba $\mathrm{T}$ de student, previa verificación de supuesto de normalidad y diferencia de proporciones usando la prueba de Ji cuadrado. Para ambos, se usó como nivel de significancia P menor a $0,05 \%$ y nivel de confianza del $95 \%$.

\section{Consideraciones éticas}

Se tuvo en cuenta la Resolución No. 008430 de 1993, del Ministerio de Salud, con una participación voluntaria, diligenciamiento de consentimiento informado por parte de los padres y asentimiento informado por parte de los adolescentes.

\section{RESULTADOS}

En la Tabla 1 se observan las variables sociodemográficas de 538 adolescentes, con una edad mínima de 9 años, máxima de 19 años y una edad promedio de 13,5 años. Curso escolar de cuarto de primaria a grado once, con $86,4 \%$ de sexto a grado decimo. En seguridad social se encontró que 114 adolescentes $(21,1 \%)$ no conocían su entidad de aseguramiento. Las 3 EPS más frecuentes fueron Saludcoop con 84 adolescentes $(15,6 \%$ ), Famisanar con 75 adolescentes $(13,9 \%)$ y Coomeva con 56 adolescentes (10,4 \%). Las demás EPS ocuparon porcentajes muy bajos. Un total de $31 \%$ pertenecían al sector público rural, 27 $\%$ al privado urbano, $25 \%$ al público urbano y $17 \%$ al privado rural.

De los 538 adolescentes encuestados, 218 (40,5\%) fueron positivos para síntomas sugestivos de ansiedad y/o depresión y 320 (59,5\%) fueron negativos. De los adolescentes que resultaron positivos, $152(28,3 \%)$ tuvieron puntaje positivo para síntomas sugestivos de ansiedad exclusivamente, 18 (3,3\%) para síntomas depresivos exclusivamente, y 48 (8,9\%) con síntomas mixtos (ansiedad y depresión). 
Tabla 1. Distribución por curso escolar, EPS y género

\begin{tabular}{|c|c|c|}
\hline Variable & Frecuencia & $\%$ \\
\hline \multicolumn{3}{|c|}{ Distribución por género } \\
\hline Femenino & 235 & 44 \\
\hline Masculino & 303 & 56 \\
\hline \multicolumn{3}{|c|}{ Distribución por curso } \\
\hline Cuarto & 16 & 3,0 \\
\hline Quinto & 19 & 3,5 \\
\hline Sexto & 117 & 21,7 \\
\hline Séptimo & 74 & 13,8 \\
\hline Octavo & 120 & 22,3 \\
\hline Noveno & 78 & 14,5 \\
\hline Decimo & 76 & 14,1 \\
\hline \multicolumn{3}{|l|}{ Distribución EPS } \\
\hline Once & 38 & 7,1 \\
\hline NS/NR & 114 & 21,2 \\
\hline Cafam & 2 & 0,4 \\
\hline Cafesalud & 7 & 1,3 \\
\hline Colmedica & 2 & 0,4 \\
\hline Colsubsidio & 2 & 0,4 \\
\hline Compensar & 41 & 7,6 \\
\hline Convida & 16 & 3,0 \\
\hline Coomeva & 56 & 10,4 \\
\hline Cruz blanca & 1 & 0,2 \\
\hline ETB & 1 & 0,2 \\
\hline Ecoopsos & 15 & 2,8 \\
\hline Famisanar & 75 & 13,9 \\
\hline Fuerza aérea & 1 & 0,2 \\
\hline Humana vivir & 11 & 2,0 \\
\hline Med. Asociados & 5 & 0,9 \\
\hline Nueva EPS & 28 & 5,2 \\
\hline Policía & 4 & 0,7 \\
\hline Salud coop & 84 & 15,6 \\
\hline Salud total & 26 & 4,8 \\
\hline Salud vida & 6 & 1,1 \\
\hline Sanitas & 33 & 6,1 \\
\hline Sisben & 1 & 0,2 \\
\hline Sura & 7 & 1,3 \\
\hline
\end{tabular}

Los síntomas ansiosos fueron más frecuentes en las mujeres y los síntomas depresivos y mixtos se presentaron con más frecuencia en hombres. De los resultados positivos para sintomas sugestivos de ansiedad, depresión o trastorno mixto en cada uno de los sectores, se encontró predominio de síntomas ansiosos en todos los sectores, seguido por sintomas mixtos y finalmente sintomas depresivos.

En la Tabla 2 se observa el promedio de puntuación en la escala SCARED (síntomas sugestivos de ansiedad) cruzado con las variables de sexo, presencia o no de síntomas sugestivos de depresión, pertenecer o 
no a colegio público y pertenecer o no a colegio urbano. Se encontraron diferencias significativas importantes en la mayoría de las variables. Hay diferencia significativa $(\mathrm{p}=0.0002)$ entre sexo y síntomas sugestivos de ansiedad, encontrando que las mujeres son más ansiosas que los hombres. Adicionalmente se encontró que si hay depresión se tiene la tendencia a estar más ansioso con un valor de $\mathrm{P}=0.0000$. Existe diferencia significativa $(\mathrm{p}=0.0008)$ entre pertenecer a colegio público y la presencia de ansiedad; es decir si el adolescente pertenece a colegio público se tiene la tendencia a estar más ansioso. Finalmente no se encontró diferencia significativa $(\mathrm{p}=$ 0.9728 ) entre pertenecer a colegio urbano y tener ansiedad.

Tabla 2. Análisis bivariado con respecto a promedios del SCARED

\begin{tabular}{|c|c|c|c|c|c|}
\hline \multicolumn{2}{|c|}{ Variable } & OBSER & X SCARED & IC $95 \%$ & $\mathrm{P}$ \\
\hline Sexo & $\begin{array}{l}\text { Masculino } \\
\text { Femenino }\end{array}$ & $\begin{array}{l}303 \\
235\end{array}$ & $\begin{array}{l}20.68 \\
24.27\end{array}$ & $\begin{array}{l}19,39-21-98 \\
22,92-25-63\end{array}$ & 0.0002 \\
\hline Depresión & $\begin{array}{l}\text { No } \\
\text { Si }\end{array}$ & $\begin{array}{c}472 \\
66\end{array}$ & $\begin{array}{l}20.90 \\
31.90\end{array}$ & $\begin{array}{l}19,96-21,84 \\
28,95-34,86\end{array}$ & 0.0000 \\
\hline Público & $\begin{array}{l}\text { No } \\
\text { Si }\end{array}$ & $\begin{array}{l}235 \\
303\end{array}$ & $\begin{array}{l}20.43 \\
23.66\end{array}$ & $\begin{array}{l}18,98-21,88 \\
22,43-24,90\end{array}$ & 0.0008 \\
\hline Urbano & $\begin{array}{l}\text { No } \\
\mathrm{Si}\end{array}$ & $\begin{array}{l}259 \\
279 \\
\end{array}$ & $\begin{array}{l}22.23 \\
22.27 \\
\end{array}$ & $\begin{array}{l}20,91-23,56 \\
20,91-23,63 \\
\end{array}$ & 0.9728 \\
\hline
\end{tabular}

En la Tabla 3 se muestra la diferencia de proporciones del promedio de puntuación en la escala CDI (síntomas sugestivos de depresión) cruzado con las variables de sexo, presencia o no de síntomas sugestivos de ansiedad, pertenecer o no a colegio público y pertenecer o no a colegio urbano. En el promedio de esta escala solo se encontraron diferencias significativas $(\mathrm{p}=0.0000)$ entre tener síntomas sugestivos de ansiedad y tener síntomas sugestivos de depresión; si hay síntomas de ansiedad se tiende a la depresión y si hay síntomas depresivos se tiende a la ansiedad, como se pudo ver en el análisis anterior. En cuanto al sexo, a pesar de tener mayor porcentaje de hombres con síntomas depresivos, no hay diferencias significativas $(\mathrm{p}=0.9128)$ entre sexos con síntomas sugestivos de depresión, es decir puede variar según la población.

En la Tabla 4 pueden observarse las diferencias de proporciones encontradas en los adolescentes con síntomas sugestivos de trastorno mixto, cruzado con las diferentes variables (sexo, colegio rural o urbano, colegio público o privado). En este análisis no se encontraron diferencias significativas en ninguna de las variables. En cuanto a la comorbilidad heterotípica (presencia de síntomas sugestivos de ansiedad y depresión al mismo tiempo) se encontró una comorbilidad del $72,7 \%$ en niños con 
riesgo de depresión y del $24 \%$ en niños con riesgo de ansiedad, lo que se ve reflejado en la literatura, pues son trastornos que tienden a coexistir.

Tabla 3. Análisis bivariado con respecto a promedios del CDI

\begin{tabular}{|c|c|c|c|c|c|}
\hline \multicolumn{2}{|c|}{ Variable } & OBSER & XCDI & IC $95 \%$ & $P$ \\
\hline \multirow{2}{*}{ Sexo } & Masculino & 303 & 10.72 & $10,02-11,43$ & \multirow{2}{*}{0.9128} \\
\hline & Femenino & 235 & 10.79 & $9,90-11,67$ & \\
\hline \multirow{2}{*}{ Ansiedad } & No & 338 & 15.34 & $14,70-15,99$ & \multirow{2}{*}{0.0000} \\
\hline & Si & 200 & 33.93 & $32,85-35,00$ & \\
\hline \multirow{2}{*}{ Publico } & No & 235 & 10.30 & $9,50-11,10$ & \multirow{2}{*}{0.1548} \\
\hline & Si & 303 & 11.10 & $10,34-11,86$ & \\
\hline \multirow{2}{*}{ Urbano } & No & 259 & 10.75 & $9,95-11,54$ & \multirow{2}{*}{0.9901} \\
\hline & $\mathrm{Si}$ & 279 & 10.75 & $9,98-11,53$ & \\
\hline
\end{tabular}

Tabla 4. Análisis bivariado con respecto a proporciones de síntomas sugestivos de trastorno mixto

\begin{tabular}{|c|c|c|c|c|}
\hline \multicolumn{2}{|c|}{ Variable } & \multicolumn{2}{|c|}{ Trastorno Mixto } & \multirow[t]{2}{*}{$P$} \\
\hline & & No & $\mathrm{Si}$ & \\
\hline \multirow{2}{*}{ Sexo } & Masculino & $0.51(0.47-0.55)$ & $0.048(0.03-0.06)$ & \multirow{2}{*}{0.7496} \\
\hline & Femenino & $0.39(0.35-0.43)$ & $0.040(0.02-0.06)$ & \\
\hline \multirow{2}{*}{ Publico } & No & $0.40(0.36-0.44)$ & $0.03(0.02-0.05)$ & \multirow{2}{*}{0.3598} \\
\hline & $\mathrm{Si}$ & $0.50(0.46-0.44)$ & $0.55(0.03-0.07)$ & \\
\hline \multirow{2}{*}{ Urbano } & No & $0.43(0.39-0.47)$ & $0.46(0.03-0.06)$ & \multirow{2}{*}{0.5620} \\
\hline & $\mathrm{Si}$ & $0.47(0.43-0.51)$ & $0.51(0.47-0.56)$ & \\
\hline
\end{tabular}

\section{DISCUSION}

Los síntomas ansiosos y depresivos tienen una gran frecuencia en la población infantil y adolescente. Esta condición se ha estudiado a profundidad en las últimas décadas y se ha incrementado la preocupación por parte de los entes de salud debido a las alarmantes cifras de prevalencia y cronicidad que parecen caracterizar, en la actualidad, a la manifestación de trastornos de ansiedad y/o depresión en la edad pediátrica $(17,18)$.

Los estudios realizados en la población general han logrado mejorar de forma considerable el conocimiento sobre la epidemiologia y clasificación de los trastornos mentales, aunque existe también un subregistro de estas patologías y es por esto que los estudios de prevalencia de estas condiciones son importantes en nuestra comunidad, para caracterizar a la población y lograr desarrollar programas de promoción y prevención de la salud mental (18-20). En este contexto, se formuló y se llevó a cabo este proyecto de investigación, obteniéndose resultados que corroboraron los porcentajes de prevalencia de estos síntomas, los cuales son similares a los reportados en la literatura mundial (18-20). 
De los 538 adolescentes estudiados se encontraron 218 resultados positivos para síntomas sugestivos de ansiedad y/o depresión, lo que corresponde a un $40,5 \%$ de la población estudiada, proporción importante y la cual es elevada si se compara con los datos globales de prevalencia de enfermedad mental en la población pediátrica (10-20 \%) (17). Esta diferencia se podría explicar porque al aplicar las escalas se detectan los individuos con síntomas sugestivos de ansiedad y/o depresión que podrían ser circunstanciales o secundarios a una situación estresante transitoria y no necesariamente corresponder a un trastorno, para lo cual se requeriría confirmar el diagnóstico con una entrevista psiquiátrica a profundidad (15).

De los 538 adolescentes encuestados, se encontró que el mayor porcentaje estaba dado por síntomas sugestivos de ansiedad con un $37 \%$, luego síntomas sugestivos de depresión con un $11 \%$, lo cual corresponde a lo encontrado en la literatura para sintomatología ansiosa y depresiva en población general infantil que reporta puede encontrarse entre el 2,6 \% y $41,2 \%$ para ansiedad y entre $6,2 \%$ y $10,6 \%$ para depresión $(21,22,23)$.

La prevalencia de síntomas depresivos encontrada en éste estudio fue similar a varios estudios realizados en Colombia, entre ellos uno realizado en Bucaramanga (2004 que reportó una prevalencia del 9,2 \% en escolares pero de una edad inferior a la de éste estudio (8-11años), y otro realizado en un municipio del oriente Antioqueño (La Ceja) utilizando el CDI, donde encontraron una prevalencia de $25,2 \%$; porcentaje muy superior a lo encontrado en nuestro estudio y a lo reportado en la literatura mundial (20). En cuanto al género, se encontró que, a diferencia de lo encontrado en el estudio de Bucaramanga, los hombres presentaron una prevalencia mayor de síntomas depresivos $6,9 \%$, en comparación con las mujeres 5,4 $\%$, pero se debe tener en cuenta que las edades del estudio también eran diferentes (18). Sin embargo, en el estudio realizado en La Ceja Antioquía, encontraron una prevalencia similar, con un 14,4 \% de síntomas sugestivos de depresión en hombres versus $10,8 \%$ en mujeres, con mayor prevalencia en los hombres (20). En cuanto a la presencia de síntomas sugestivos de ansiedad y relación con el género, se encontró que es significativamente mayor la presencia de ansiedad en mujeres que en hombres, lo cual concuerda con los hallazgos reportados por la literatura en general (20). Para síntomas depresivos y síntomas mixtos, a pesar de predominar estos trastornos en los hombres, no se encontró diferencia significativa en cuanto 
a la frecuencia según el sexo para estos, lo cual contrasta con otros estudios en donde es mayor la frecuencia de depresión en mujeres pero de mayor edad (18).

Se concluye entonces que los síntomas ansiosos y depresivos son frecuentes en la población de adolescentes, por lo cual se hace indispensable sospecharlos y detectarlos de manera temprana para que reciban una atención oportuna, implementando medidas de educación a padres, maestros y orientadores. La prevención radica en fortalecer los factores protectores como autoestima y resiliencia para evitar la aparición de estos trastornos. El futuro de la salud de nuestras comunidades, no depende solo de nuestra capacidad de enfrentar las enfermedades agudas, sino, sobre todo, de las respuestas que podamos implementar ante los desafíos de un mundo cambiante; y la irrupción de las enfermedades crónicas, en especial las que afectan el comportamiento, la salud mental y la calidad de vida de nuestros niños, las cuales de no ser tratadas a tiempo pueden causar comorbilidad y complicaciones, como el suicidio, dejando secuelas tanto para la familia como para la comunidad

\section{REFERENCIAS}

1. De Zubiría Samper M. Marco general. En: Sánchez A. Como prevenir la soledad, la depresión y el suicidio en niños y jóvenes, un manual para que sus hijos vivan mejor. Bogotá, Colombia: Aguilar Altea; 2007. p. 13-24.

2. Murray CJL, Lopez A. Progress and directions in refining the global burden of disease approach: a response to Williams. Health Economics 2000; (9):69-82.

3. Organización Mundial de la Salud (OMS). Informe mundial sobre la salud 2001. Mental Health: New Understanding, New Hope. Ginebra; 2001.

4. Organización Panamericana de la Salud: Presentación al Consejo Directivo (CD40.R19). Washington D.C; Septiembre 1997.

5. Giberti E. Políticas y niñez. Editorial Losada; 1997.

6. Eroles C, Fazzio A, Scandizzo G. Políticas públicas de Infancia [Editorial]. Espacio. Buenos Aires; 2001.

7. Christopher KV, Smith C. Anxiety disorders in the child and teen. Pediatr Clin N Am 2003; (50)1107- 1138.

8. Murray CJL, Lopez AD. Global health statistics. Cambridge, MA: Harvard School of Public Health. Organización Mundial de la Salud, Banco Mundial (Global Burden of Disease and Injury Series (II);1996.

9. Organización Mundial de la Salud (OMS).Programa de salud adolescente, familia y salud reproductiva. The Second Decade: Improving Adolescent Health and Development. Ginebra; 1998.

10. Levav I. Salud mental, prioridad en la Salud pública. Norte de Salud Mental. 2004;(19) 9-22.

11. Organización Mundial de la Salud. (OMS) Improving mother/child interaction to promote better psychosocial development in children. Ginebra. WHO/MSA/MHP/98.1.1998 
12. Sitio oficial alcaldía Chía [Internet]. Disponible en: http://www.chia.gov.co/ Consultado en Diciembre 2008.

13. Organización Mundial de la Salud (OMS). Atlas Recursos de Salud Mental en el Mundo. Ginebra; 2001.

14. Organización Mundial de la Salud (OMS). Departamento de Salud Mental y Dependencia de Substancias. Caring for Children and Adolescents with Mental Disorders: Setting WHO Directions. Ginebra; 2003.

15. Figueiras MA, Amador JA, Gómez J, Del Barrio V. Psychometric Properties children's depression Inventory in community and clinical sample. The Spanish Journal of Psychology. 2010; 13 (2) 990-999.

16. Barmier B, Brent D. Psychometric Properties of the Screen for Child Anxiety Related Emotional Disorders (SCAReD): A Replication Study. Journal of the American Academy of Child and Adolescents Psychiatry. 1999; 38 (10):1230-1236.

17. OMS. Carga de los trastornos mentales y conductuales. Capítulo 2. Informe sobre la salud en el mundo; 2001. Publicaciones Organización Mundial de la Salud 2001. Ginebra, Suiza.

18. Mantilla L, Sabalza L, Díaz L, Campo-Arias A. Prevalencia de síntomas depresivos en niños y niñas escolares de Bucaramanga, Colombia. Revista Colombiana de Psiquiatría. 2004: 33: 163-171.

19. Romero K, Canals J, Hernández-Martínez C, Jané.Balladriga M, Viñas F, DomènechLlaberia F. Comorbilidad entre los factores de ansiedad del SCARED y la sintomatología depresiva en niños de 8-12 años. Psicothema. 2010; 22, (4), 613618.

20. Vinaccia S, Gaviria A, Atehortúa L, Martínez P, Trujillo C, Quiceno J. Prevalencia de depresión en niños escolarizados entre 8 y 12 años del oriente antioqueño a partir del "Child Depression Inventory" -CDI. Perspectivas en psicología. 2006; 2(1):217-227.

21. Cartwright-Hatton S, McNicol K, Doubleday E. Anxiety in a neglected population: Prevalence of anxiety disorders in pre-adolescent children. Clinical Psychology Review, Special issue: Anxiety of Childhood and Adolescent: Challenges and opportunities. 2006; 26(7): 817-833.

22. Boyd C, Gullone E, Kostanski M, Ollendick T, Shek D. Prevalence of Anxiety and Depression in Australian Adolescents: Comparisons with worldwide data. The Journal of Genetic Psychology. 2000; 161(4):479-492.

23. Frigerio A, Pesenti S, Molteni M, Snide J, Battaglia M. Depressive symptoms as measured by the CDI in a population of northern Italian children. European Psychiatry,2001;16(1): 33-7. 